\title{
Diseño e Implementación del Programa Galicia de Reeducación de Maltratadores: Una Respuesta Psicosocial a una Necesidad Social y Penitenciaria
}

\section{Design and Implementation of the Galician Program for Batterers' Re-education: A Psychosocial Answer to a Social and Penitentiary Need}

\author{
Ramón Arce \\ Universidad de Santiago de Compostela
}

Francisca Fariña
Universidad de Vigo

\begin{abstract}
Resumen. La aprobación y entrada en vigor en España de la Ley Orgánica 1/2004, de Medidas de Protección Integral contra la Violencia de Género, el 29 de junio de 2005, ha traído consigo que un gran número de sentencias contemplen la suspensión o sustitución de la pena por programas de reeducación y tratamiento psicológico. Una revisión del estado de la literatura sobre los modelos teóricos de la violencia y la eficacia de las intervenciones con penados por violencia de género, puso de manifiesto que las intervenciones ajustadas a las necesidades y características específicas de cada penado eran las más efectivas. Para la identificación de éstas, se ha confeccionado un sistema de evaluación que se discute en este trabajo. Asimismo, la literatura también soporta la pertinencia de una intervención multimodal (cognitivo y comportamental) y multinivel (p.e., individual, grupal, red social, laboral). Adicionalmente, se discuten otros condicionantes del programa de intervención que median la eficiencia de ésta. Sobre estas premisas se confeccionó el Programa Galicia de Reeducación de Maltratadores de Género (Arce y Fariña, 2007), cuyos resultados desde su puesta en marcha en 2005 a 2010 están siendo satisfactorios.

Palabras clave: tratamiento penitenciario, maltratadores, violencia de género, reeducación, intervención psicososical.
\end{abstract}

\begin{abstract}
The introduction in Spain of the Spanish Law on Comprehensive Preventive and Protective Measures against Gender Violence, (L.O. 1/2004), has supposed that a very large number of sentenced as batterers who are eligible to parole or supervision orders as an alternative to incarceration. This paper reviews the state of the art in terms of the theoretical explanations and efficacy of the interventions, concluding that the intervention that has proven to be more effective is one driven the specific needs and characteristics of the batterer than those based on general intervention programs for all the batterers. The evaluation schedule for this aim is discussed. Other additional features of the intervention that mediate the efficiency of the outcomes are also discussed. For the treatment under these conditions, in Galician, Norwest of Spain, a psychosocial program was developed the Galician Programme for the Treatment and Re-education of Convicted Gender Aggressors (Arce and Fariña, 2007). The results of the implementation of this program from 2005 to 2010 are satisfactory.

Keywords: treatment of prisoners, batterer, domestic violence, reeducation, psychosocial intervention.
\end{abstract}

\section{De los modelos explicativos de la violencia}

El comportamiento violento, entendiendo por tal el patrón de conducta caracterizado por un comportamiento, usualmente persistente y repetitivo, en el que no se siguen las normas sociales ni se respetan los derechos de los demás (American Psychiatric Association, 2002), ha sido uno de los tópicos relacionados con el comportamiento humano que más atención ha merecido a lo largo de la historia por parte de

La correspondencia sobre este artículo puede dirigirse al primer autor al Departamento de Psicología Social. Facultad de Psicología. Universidad de Santiago de Compostela. 15872 Santiago de Compostela (A Coruña).e-mail: ramon.arce@usc.es pensadores y científicos. De hecho, se ha abordado desde múltiples perspectivas, legal, criminológica, psicológica, sociológica o médica. Si bien se ha intentado explicar el comportamiento desviado desde multitud de perspectivas, los diferentes intentos se han orientado hacia la maximización de alguno de los siguientes tres factores (sería demasiado larga la revisión de estos postulados y excedería el propósito de este trabajo, para ello véase una recopilación en Fariña y Arce, 2003): biológicos (p.e., Lombroso, los constitucionalistas, o las formulaciones de Eysenck) psicológicoindividuales (v.gr., teorías del aprendizaje social, del desarrollo cognitivo moral o de la decisión racional) y psicológico-sociales (ad exemplum, Merton, Cohen, Cloward y Ohlin, Sutherland, Hirschi). Ahora bien, 
generalmente en estas teorías la explicación que se ofrece de la delincuencia se orienta a la sobredimensionalización de una(s) variable(s) o dimensión(es) en detrimento de otras, lo cual lleva implícito que el valor de las mismas sea relativo. Además, la sobredimensionalización de una o más dimensiones en detrimento de otras, también se refleja en los tratamientos que están orientados, generalmente, bien a aislar los efectos de un único componente, bien a recurrir a una fórmula de tratamiento aplicable al conjunto del problema pero desde una única perspectiva (p.e., farmacológico, castigo y refuerzo, resocialización, barrera). En consecuencia, la adopción de estos modelos aisladamente no es operativa ni a nivel de explicación de las causas del comportamiento violento de todos los agresores, como requiere la intervención penitenciaria en violencia de género, muy especialmente con aquellos que cumplen el tratamiento en libertad, ni del tratamiento pues es evidente que no sería apropiados para todos aquellos penados a los que el modelo causal aplicado no se ajustado a su caso.

La carencia de eficacia preventiva y de tratamiento de estos modelos, motivada principalmente en el excesivo reduccionismo explicativo, dio lugar a la propuesta de hipótesis teóricas integradoras (e.g., Farrington, 1992; Feldman, 1989; Gottfredson y Hirschi, 1990). Sin embargo, la integración de los tres grupos de factores en una misma teoría no está exenta de problemas, ya que no resultaron operativas ni definidas adecuadamente al nivel de tratamiento, ni aumentaron significativamente el nivel de explicación del comportamiento desviado. Por todo ello, deberíamos admitir que, si bien el principio de parsimonia es irrenunciable, desde una óptica científica o normativa no caben, pues, aproximaciones tan solipsistas para un problema multicausal. En todo caso, de estas aproximaciones han emanado dos importantes aportaciones. La primera (i.e., Feldman, 1989) gira en torno al aprendizaje del comportamiento antisocial y prosocial. En concreto, Feldman sostiene que tanto la conducta prosocial como la desviada se aprenden de modo que el sujeto actúa en función de lo aprendido. Aparte de las limitaciones que presenta en la sistematización del aprendizaje, esta propuesta mantiene la exclusividad de un aprendizaje u otro cuando, en la realidad, los sujetos han aprendido ambos comportamientos, esto es, todos hemos aprendido comportamientos violentos y no violentos, por lo que estamos capacitados para actuar con y sin violencia. La segunda, basada en la probabilidad de riesgo, que no de la peligrosidad, es la hipótesis de riesgo/protección que se ha dirigido a perfilar las variables de riesgo y protección contra la desviación (p.e., Farrington, 1992). Los factores de riesgo vienen a ser las variables individuales y ambientales que aparecen vinculadas con el comportamiento desviado. Los factores protectores, por su parte, bien fueron definidos justamente como aquellas variables individuales y ambientales vinculadas al comportamiento prosocial
(Farrington, 1992) ${ }^{1}$, bien como aquellas que potencian las capacidades de los individuos expuestos a altos niveles de riesgo para afrontar con éxito dichas situaciones adversas $^{2}$ (Ross y Fabiano, 1985). Los modelos de riesgo han identificado como variables que actúan como facilitadoras del comportamiento criminal (Farrington, 1996; Andrews y Bonta, 2006): los factores pre- y peri-natales; hiperactividad e impulsividad; inteligencia baja y pocos conocimientos; supervisión, disciplina y actitudes parentales; hogares rotos; criminalidad parental; familias de gran tamaño; deprivación socioeconómica; influencias de los iguales; influencias escolares; influencias de la comunidad; y variables contextuales. La relación entre estos factores y el comportamiento desviado se asumió, inicialmente, que era lineal, por lo que sería suficiente tomar como factor protector el mismo que se había detectado como de riesgo, y viceversa. En consecuencia, los factores protectores serían los antagonistas de los factores de riesgo, y los de riesgo los de los protectores. Esta premisa no siempre es correcta. Así, la baja inteligencia se relaciona con el comportamiento antisocial, pero de ello no se puede inferir directamente que una inteligencia normalizada o alta proteja contra dicho comportamiento, si así fuera no se registraría la delincuencia económica, de cuello blanco o la corrupción (Bartol, 1999). Como consecuencia, Lösel y Bender (2003) revisaron los factores protectores identificando los diez siguientes: factores psicofisiológicos y biológicos; temperamento y otras características de personalidad; competencias cognitivas; apego a otros significativos; cuidado en la familia y otros contextos; rendimiento escolar; vínculo con la escuela y empleo; redes sociales y grupos de iguales; cogniciones relacionadas con uno mismo, cogniciones sociales y creencias; y factores de la comunidad y vecindario. En resumen, estas hipótesis, que únicamente tienen pretensiones descriptivas y no descriptivo-prescriptivas, como sería necesario, han dejado dos legados muy significativos para el avance en la comprensión y tratamiento del comportamiento antisocial y delictivo: éste, al igual que el prosocial, no es innato, sino que requiere de un aprendizaje, y la identificación de los factores de riesgo y protectores para su aprendizaje y generalización.

\section{De los paradigmas aplicables al comportamiento antisocial y delictivo}

Dos son los paradigmas que permiten ajustar los modelos de protección o déficit de aprendizaje y generalización del comportamiento antisocial y delictivo a

\footnotetext{
${ }^{1}$ Los datos se obtienen de estudios longitudinales, tomando el concepto de riesgo estadístico (i.e., probabilidad) que es preciso tener presente que no informa sobre los mecanismos causales subyacentes.

2 El diseño básico consiste en la comparación de muestras de desviados (v. gr., presos, reincidentes) con otros no desviados o rehabilitados
} 
la realidad del sujeto: el paradigma de déficit aditivos/acumulativos (Lösell, Kolip, y Bender, 1992) y el paradigma de no-modelo (Arce y Fariña, 1996).

Como quiera que estos factores de protección o riesgo no se dan de forma aislada, sino en combinación unos con otros, la combinación de los factores de riesgo ha dado lugar a los modelos de vulnerabilidad o de déficit de destrezas (p.e., Ross y Fabiano, 1985; McGuire, 2000; Werner, 1986, Zubin, 1989) en tanto la de los factores de protección a los de competencia (v.gr., Lösell et al., 1992; Wallston, 1992). Se entiende por modelos de vulnerabilidad o de déficit de destrezas un conjunto de variables que se dan juntas y facilitan el comportamiento antisocial y delictivo. Por el contrario, un modelo de competencia se obtiene de la unión de variables de protección que pueden sumar sus efectos. Los modelos de competencia social que se han formulado bajo este soporte han sido muy numerosos. Así, D’Zurilla (1986), entiende la competencia social como un amplio rango de habilidades y estrategias de afrontamiento; Peterson y Leigh (1990) como la conjunción de los procesos atribucionales, las habilidades interpersonales y la empatía; Garrido y López (1995) como el resultado de destrezas cognitivas (p.e., razonamiento, pensamiento, resolución de problemas), habilidades sociales, valores y control emocional; entre muchos otros (véase para una revisión, López, Garrido, y Ross, 2001). En todo caso, todas estas propuestas agrupan un rango de variables cognitivas, sociales o ambas para explicar, en último término, la competencia cognitivo-social o no del individuo frente al comportamiento antisocial o delictivo. Por tanto, el objeto último de análisis es la competencia o incompetencia del individuo en los contextos de riesgo de desviación.

El paradigma de no-modelo (Arce y Fariña, 1996), por su parte, propone que no es posible reducir el comportamiento humano, entre el comportamiento antisocial y delictivo, a un único modelo explicativo, sino que cada contexto y cada caso precisa de la asunción de un modelo específico que se ajuste al mismo. Para ello, se distingue, en consonancia de la literatura, entre causas facilitadoras del comportamiento antisocial, los denominados factores de riesgo que constituyen las causas primarias, que son estáticos para el agresor (en nuestro caso, el maltratador), o sea, no puede intervenir sobre ellos, y los efectos indirectos o secundarios para el agresor de estas causas primarias (p.e., carencias en el aprendizaje) que pueden llegar a ser factores dinámicos para el agresor, o sea, que se puede actuar sobre ellos. De ahí, que agresores en contextos de riesgo puedan resultar prosociales, y viceversa. Así, cuando el origen del comportamiento delictivo radica en la socialización en una comunidad/barrio marginal o en una familia desestructurada, sólo hemos reconocido la causa primaria del comportamiento antisocial, restando aún por identificar los efectos secundarios o indirectos sobre la biología, aprendizaje, cogniciones y comportamiento del individuo, entre otros. En el caso de un ambiente familiar de riesgo, nosotros (Arce, Seijo, Fariña, y Novo, 2004) hemos observado que este factor llevaba a que agresores criados en una familia de riesgo manifiesten, además de más comportamientos antisociales, inadaptación en todos los niveles, y exhiban desajustes en el autoconcepto, habilidades sociales, en el uso recurrente a estrategias de afrontamiento de huida intrapunitiva y en un perfil clínico tendente a la patología (v.gr., hostilidad, depresión, ideación paranoide). En lo tocante al factor de riesgo de la comunidad/barrio, hemos encontrado (Fariña, Arce, y Seijo, 2005) que los agresores socializados en una comunidad/barrio de alto riesgo emitían más comportamientos desviados, tanto antisociales como delictivos, que los educados en un barrio de bajo riesgo, de lo que se deduce que éstos presentan una mayor tendencia al desarrollo de una carrera criminal bien sea de curso vital bien sea limitada (Moffitt, 1993). La comunidad, como factor estático para el individuo, esto es, sobre el que éste no puede actuar, llevaba a que los agresores socializados en una comunidad de riesgo evidenciaran como efectos indirectos un déficit significativo en el autoconcepto, en los procesos atributivos, en las estrategias de afrontamiento y en la inteligencia emocional, convirtiéndolos en sujetos con menor competencia social; esto es, más vulnerables (D’Zurilla, 1986; Ross y Fabiano, 1985; Garrido y López, 1995; Peterson y Leigh, 1990). En suma, la socialización en una comunidad/barrio de riesgo no sólo facilita la adquisición de comportamiento antisocial, sino que también inhibe el desarrollo de los factores cognitivo-sociales protectores (i.e., atribución interna, estilo de afrontamiento positivo, autoconcepto positivo), y la menor competencia social, que se ha mostrado invariablemente relacionada con el riesgo de carrera criminal (Andrews y Bonta, 2006; McGuire, 2000). Complementariamente, este factor estático puede actuar como protector cuando facilita el aprendizaje prosocial a través de la transmisión de destrezas propias de la competencia social tal como verificamos que ocurría en las comunidades/ barrios de bajo riesgo. En consecuencia, este modelo presenta dos lecturas: una de vulnerabilidad, en el grupo de riesgo, y otra de protección, en el de no riesgo. Pero los modelos obtenidos de los datos aplicables a la comunidad y familia, que no son homogéneos entre sí, tampoco son generalizables a todos los factores de protección o riesgo y mucho menos a cada caso particular. De hecho, en los contextos de riesgo se encuentran individuos prosociales, y viceversa. Aún es más, cada individuo específico va a precisar el ajuste del modelo a sus déficits o efectos indirectos específi$\cos$. En todo caso, las carencias se han de acomodar, de acuerdo con los hallazgos de Arce, Fariña, Seijo, Novo, y Vázquez (2005), a los efectos en el área biológica (patología e inteligencia emocional), psicológico (v.gr., carencia de habilidades sociales e interpersonales; habilidades cognitivas tal como empatía, razo- 
namiento, pensamiento o resolución de problemas; carencia de un desarrollo moral; recurso a estrategias de afrontamiento negativas), familiar (familia de riesgo), académico/laboral (inadaptación escolar) y sociocomunitaria (p.e., red social incompetente). En otras palabras, complementando al paradigma de déficit aditivos, los hallazgos empíricos han puesto de manifiesto que los efectos indirectos van más allá del área individual, el objeto de intervención desde el paradigma aditivo, alcanzado a las áreas biológica, familiar, académico-laboral y socio-comunitaria. Como se habrá observado, se han ajustado los hallazgos a 5 áreas, en consonancia con el Eje V del DSM-IV en el que se valoran daños (American Psychiatric Association, 2002), frente a las tres tradicionales (biológica, psicológica y socio-comunitaria) porque esta división refleja mejor las áreas en las que se valora el daño psicológico y que, por tanto, han de constituir el objeto central de una intervención psicológica diferenciada.

\section{De las características sociolegales específicas de la intervención con penados por violencia de género en régimen de libertad}

El comportamiento delictivo contra las personas y, más específicamente, contra las mujeres, supone la ejecución por parte del agresor de actos de violencia física y psicológica, incluidas las agresiones a la libertad sexual, las amenazas, las coacciones o la privación arbitraria de libertad (art. 1.3 de la L.O. 1/2004), que conllevan en la víctimas secuelas físicas o mentales, sufrimiento emocional, pérdida o daño material, o un menoscabo importante en sus derechos (United Nations, 1988). Este tipo de delincuentes responden fundamentalmente a dos patrones o tipologías: el violento en general y el violento sólo en el ámbito de la pareja (Holtzworth-Munroe y Stuart, 2004); es decir, la primera tipología se circunscribe al delincuente antisocial, en tanto la segunda se relaciona con agresores selectivos; en concreto, dirigido a las mujeres con las que mantiene una relación de pareja. Los tratamientos a administrar, por las diferencias entre éstos, han de implicar contenidos distintos (Loinaz, Torrubia, Echeburúa, Navarro, y Fernández, 2009). Por su parte, las condenas a las que son sometidos por los juzgados y tribunales agrupan a los penados en torno a dos tipos de tratamiento cualitativamente distintos: ejecutado en un centro penitenciario en régimen cerrado y ejecutado en libertad. Es precisamente este último tipo de condena el preponderante en casos de violencia de género. Los penados que cumplen la condena en condiciones de libertad tienen una serie de características que es preciso tener en mente a la hora de su tratamiento: son primarios; han cometido delitos contra las personas en el ámbito privado; las agresiones se derivan del ejercicio del poder asociado al géne- ro masculino; la violencia ejercitada puede ser de muy diverso tipo ( $v . g r$., física, psíquica, sexual, amenazas, coacciones, privación de la libertad); la violencia no tiene porqué estar presente en otros ámbitos; y la alta probabilidad de reagresiones (violencia continuada) y de recaídas (Echeburúa, Fernández-Montalvo, y Corral, 2008; Ministerio de Igualdad, 2009). Adicionalmente, se espera un cierto índice de patología dual, esto es, la combinación de violencia de género con otras patologías como alcoholismo, drogodependencia, ludopatía, daños en las funciones neuropsicológicas o psicopatología (Bersani y Chen, 1988; Smith, 1993; Wallace, 1996). Los datos de nuestro programa cifran estas contingencias, como trastorno primario, en adicciones (alcoholismo, drogodependencia y/o ludopatía) en torno a un $25 \%$; a un $3 \%$, los trastornos mentales incapacitantes; y a un poco más del $1 \%$, las carencias en las funciones neuropsicológicas básicas que condicionan, cuando no imposibilitan, el tratamiento (Fariña, Arce, y Novo, 2008). En consecuencia, los planes de intervención en estas condiciones y sobre este tipo de penados han de ser ajustados rigurosamente a cada caso, estar muy controlados y ser altamente efectivos porque el riesgo, al implementarse en condiciones de libertad, es sumamente elevado. Son, por tanto, improcedentes las intervenciones generalistas, incompletas, mal diseñadas o parciales, con cronologías incoherentes o inconsistentes, no sólo por la pérdida de efectividad, sino también porque no afrontan el riesgo de recaídas durante el tratamiento. No en vano, los resultados del Programa Galicia de Reeducación de Maltratadores de Género avalan que más de la mitad de los penados, el $52 \%$, en cumplimento de la condena informan que tienen una nueva pareja (a esta cifra habría que añadir la convivencia oculta con la propia víctima, a que en muchas ocasiones son parejas inestables, a la tendencia observada que presentan los agresores a ocultar este dato, para evitarse problemas en la ejecución del tratamiento, etc.). Es por ello que todo plan de intervención requiere de un modelo que lo sustente no bastando sumar un conjunto de intervenciones más o menos coherentes entre sí, e incluso muy semejantes a otros modelos de intervención eficaces; temporalizaciones arbitrarias en la ejecución de la intervención; o no sujetas adecuadamente a un desarrollo consistente en la ejecución del programa. Sin embargo, la gran mayoría de las intervenciones penitenciarias están tasadas y son repetitivas para todos los penados. Mutantis mutandis, vendría a ser como si a los enfermos de diversas dolencias físicas se les administrase siempre, con independencia de la dolencia y de su etiología, el mismo tratamiento, si la pautación del tratamiento no se ajustara a las características morfológicas del enfermo, o si la posología no siguiera una pauta definida y comprobada. Al respecto, McGuire, Mason, y O'Kane (2000) relacionaron los elementos clave sobre los que debe pivotar la intervención: 
a) Los programas de intervención han de responder a un modelo explícito y bien articulado de las causas, a tiempo que han de estar sostenidos por evidencia científica contrastada.

b) Un reconocimiento de la importancia de la evaluación del riesgo que, en el caso que nos ocupa, el tratamiento de la violencia de género y en condiciones de libertad, es crítico. Por ello, esta evaluación del riego ha de llevarse a cabo de forma continuada.

c) La evaluación de los factores dinámicos de riesgo.

d) La aplicación de técnicas y métodos que impliquen un aprendizaje participativo, activo y focalizado (principio de responsabilidad), así como aquellas que se ha contrastado que facilitan el cambio. A todo esto, le añadimos que el objeto de la intervención ha de ajustarse a los déficits o características propias de cada penado.

e) Concreción de objetivos claros, bien estructurados y que respondan a las necesidades particulares del penado.

f) La aplicación de una aproximación multimodal, a lo que añadimos que ha de ser también multinivel.

g) Que la duración de la intervención se ajuste a las necesidades y evolución en el tratamiento de cada penado. Por ello, se ha de llevar a cabo una evaluación continua del progreso del penado.

h) La implementación de la intervención por personal especializado y entrenado, que disponga de los recursos adecuados, que la ratio técnicopenado sea tal que permita la implicación del primero; y que el técnico esté a tarea única (no puede participar en diferentes programas al mismo tiempo con objetivos, técnicas y contenidos distintos ya que lo desfocalizan).

Antes de entrar de lleno en la planificación de una intervención, es necesario establecer las líneas base y tomar en consideración si es factible la intervención. El punto de partida pasa por someter a contraste la máxima nada funciona. La primera de las intervenciones a analizar es la condena judicial. No en vano, se han formulado leyes en muchos países, para afrontar desde una óptica legal la violencia de género y doméstica, basadas todas ellas en una intervención integral en la que la condena del agresor es un punto central. De hecho, sin agresor, no hay violencia. Así, el internamiento en prisión en régimen cerrado elimina toda posibilidad de reincidencia. Ahora bien, la intervención penitenciaria está delimitada por un tiempo definido en sentencia judicial por lo que es necesario conocer los efectos post-condena. No ocurre lo mismo con las condenas que se ejecutan en libertad en las que el riesgo de reincidencia está muy presente por un doble motivo. El penado puede acceder a la víctima u otras mujeres, especialmente aquella con la que mantiene una relación. La evidencia empírica desafortunadamente ha puesto de manifiesto que la condena por violencia de género sólo reduce muy modestamente la reincidencia (Ventura y Davis, 2005). Dado que sin maltratadores no hay violencia de género y que el predictor más potente de un agresor son los antecedentes como tal (e.g., Bonta, Law, y Hanson, 1998; Gondolf y White, 2001; Puffet y Gavin, 2004), es preciso explorar otras intervenciones complementarias a la condena. En suma, la condena no es muy efectiva en la erradicación de la violencia contra las mujeres, pero sí que es una buena oportunidad para intervenir con el maltratador. La literatura nos advierte que los programas de intervención con agresores primarios de violencia doméstica no sólo son posibles (Echeburúa, Corral, FernándezMontalvo, y Amor, 2004), sino también efectivos tanto en la adquisición de destrezas (i.e., control emocional, cambio actitudinal), disminución de la frecuencia de conductas de maltrato (i.e., Echeburúa y Fernández-Montalvo, 1997, 1998; Taylor, Davis, y Maxwell, 2001); como en la prevención de recaídas (v.gr., Carney y Buttell, 2004; Fracher, 2003; Gondolf, 2004). En una revisión de los programas de intervención con todo tipo de agresores, Beelmann y Lösel (2006) encontraron apoyo a la pertinencia de los programas de entrenamiento multimodales (esto es, cognitivo-conductuales) en competencia social, especialmente en poblaciones de riesgo. No obstante, la efectividad de la aplicación generalizada de este tipo de programas con maltratadores y los derivados de la propuesta Duluth es muy limitada (esto es, es significativa, pero con tamaños del efecto realmente bajos) (Babcok, Green, y Rubie, 2004), e incluso pueden resultar inconvenientes para aquellos penados a los que no se ajusta la intervención a sus necesidades, lo que es bastante probable (Bowen, Gilchrist, y Beech, 2008). Así, se ha propuesto que se ajusten los tratamientos, cuando menos, a tipologías de maltratadores (Holtzworth-Munroe y Stuart, 2004), especialmente los grupos de maltratadores sólo violentos de género y maltratadores violentos en general (Loinaz et al., 2009). No en vano, el ajuste de la intervención a las necesidades y circunstancias de los penados, potencia la eficacia del tratamiento (HoltzworthMunroe, Meehan, Herron, Rehman, y Stuart, 2000). Ahora bien, las tipologías de maltratadores, si bien permiten ciertas agrupaciones de agresores para agruparlos en el tratamiento, éstas serían interminables para llegar a todos los maltratadores, al tiempo que no responderían a las peculiaridades específicas de cada penado. Así pues, procede ajustar el tratamiento a cada penado, constituyéndose grupos de tratamiento post hoc en función de la agrupación de los mismos por la homogeneidad en las características y necesidades registradas en la evaluación. En resumen, la máxima de nada funciona afortunadamente no es sostenible, sino que hay procedimientos que mejoran la eficacia de la intervención, pero no todos por igual, ni 
pueden generalizarse a todos los casos. En consecuencia, no son adecuadas las fórmulas basadas en una intervención generalizada, sino que es necesario concretar planes de intervención acomodados a cada penado y sus circunstancias.

\section{De la necesidad de una intervención integral}

A la vista de los planteamientos anteriores cabe preguntarnos si cabe o no una intervención. Las revisiones de la literatura informan sistemáticamente de la efectividad de las intervenciones bien planificadas y ejecutadas (i.e., Beelmann y Lösel, 2006; Farrington, 2003). No obstante, la efectividad varía de un tipo de programas a otros, e incluso pueden no ser efectivos (ha de tenerse presente que los datos inconvenientes o que no confirman efectos raramente se publican) (McGuire, Mason, y O'Kane, 2000). Por ello y partiendo de que la intervención es viable, es preciso resolver cuál ha de ser el objeto de la misma así como su alcance. Una segunda pregunta que surge es cuestionarnos cuál ha de ser el objeto y alcance de la misma. Desde la perspectiva de los modelos de vulnerabilidad/competencia, el tratamiento requiere de una aproximación multimodal, esto es, cognitivo (v.gr., cambio actitudinal, entrenamiento en pensmaiento) y comportamental (p.e., ensayo conductual), ya que se entiende que los dos modos de actuación son complementarios. No en vano, si sólo se interviene sobre el comportamiento estaríamos ante los modelos de tratamiento conductuales basados en el castigo/refuerzo o la imitación que bien es sabido que implican la generalización de lo aprendido, ya que realmente no enseñan. Por su parte, una intervención dirigida únicamente a las cogniciones del individuo presenta dificultades de actuación debido a que puede no poseer el repertorio conductual esperado, no tener habilidades sociales para actuar conforme a las cogniciones, o no generalizar el comportamiento adaptativo a los contextos esperados. En consecuencia, una intervención multimodal es más robusta y eficaz que una unimodal. No obstante, este tipo de intervención sólo tiene por objeto el individuo, tomando los factores biológicos y los sociocomunitarios en los que se desenvuelve éste como factores estáticos, o sea, que no son o no pueden ser objeto de intervención. Ahora bien, Farrington (2003), en una revisión sobre el tratamiento del comportamiento antisocial, observó que es viable y productiva una intervención sobre los aspectos biológicos, de socialización familiar, educativos y socio-comunitarios que envuelven al individuo y que, en buena medida, condicionan o determinan su comportamiento. En suma, estos factores estáticos para el individuo (esto es, no puede intervenir sobre ellos) pueden ser dinámicos (o sea, objeto de intervención positiva) en la intervención técnico-profesional. Al respecto, el modelo de nomodelo conduce a la adopción al unísono de una apro- ximación de tratamiento multimodal, al igual que los modelos de competencia/vulnerabilidad, y multinivel, lo que permite abordar esos factores estáticos en los modelos de competencia cognitivo-social como dinámicos, es decir, sujetos a intervención. Por multinivel se entiende (Fariña, Arce, y Novo, 2008a) que la intervención no sólo debe ceñirse al sujeto de riesgo, como se ha llevado a cabo casi exclusivamente, sino que también es preciso que abarque las otras áreas que median el comportamiento del individuo. La asunción de soluciones parciales lograría, sobre la base de un modelo aditivo o acumulativo, reducir las probabilidades de recaída, pero los logros obviamente serán menores que los de un abordaje integral. Así para el caso que nos ocupa, condenados por violencia de género, se deriva una intervención en la área individual (v.gr., adquisición de competencia social), la área biológica (p.e., inteligencia emocional), familiar (i.e., escuela de padres), educativa/formativa ( $v . g r$., capacitación laboral) y área socio-comunitaria (e.g., red social, ambiente social de convivencia). Por ejemplo, un condenado por violencia de género al que se le aplica una intervención multimodal bajo unas condiciones que faciliten la creación de una red informal de maltratadores fuera del contexto específico de la implementación del tratamiento, tiene una mayor probabilidad de reincidencia que aquel no expuesto a una red de este tipo (Beleña y Báguena, 1993). Lo mismo ocurriría si no posee unas capacidades para la inserción laboral y, por lo tanto, no es capaz de obtener los recursos para su subsistencia. Asimismo, si vuelve a un ambiente socio-comunitario antisocial (p.e., a una comunidad con contenidos y actitudes sexistas) o incompetente socialmente, las probabilidades de reincidencia son más elevadas (Gracia, Herrero, Lila, y Fuente, 2009). En consecuencia, desde el modelo de no-modelo es preciso proceder a una evaluación de las carencias del individuo en los diferentes niveles de actuación del mismo para ajustar la intervención a estas carencias y niveles. Así, el modelo explicativo y, por extensión, la intervención ha de ser ajustado a cada individuo y a sus circunstancias. Para los planes de intervención, esta solución presupone adoptar un programa de intervención con implicaciones en las diferentes áreas en las que se observen carencias o disfunciones: individual, donde se recogen las intervenciones a llevar a cabo, directamente con el penado; familiar en la que se incluyen las actuaciones en el ámbito familiar para la reeducación del maltratador, con especial incidencia en aquellos casos en los que tiene una nueva pareja; socio-comunitaria que engloba las acciones a tomar sobre el ambiente en que se desenvuelve encaminadas a su reeducación, rehabilitación y reinserción; y, finalmente, laboral, donde se aglutinan las medidas orientadas a la (re)inserción social (cuando está integrado laboralmente, el plan de intervención ha de ser escrupulosamente respetuoso con no interferir de ningún modo en la estabilidad laboral, tanto por 
las implicaciones que tiene para la inserción social del propio penado, como por las obligaciones con su expareja e hijos que ha de mantener). La asunción de realidades de tratamiento menos desarrolladas conlleva indefectiblemente a una menor garantía de éxito del mismo y, por ende, a una mayor probabilidad de reincidencia en el maltratado tras la intervención.

\section{De la evaluación del penado}

Para concretar una intervención individualizada y programada para cada penado que potencie su reeducación y reinserción es necesario identificar todas las disfunciones que puedan mediar la eficacia del tratamiento, las necesidades, carencias y déficits asociados a la violencia de género. Sucintamente, la literatura $(\mathrm{ad}$ exemplum, Bersani y Chen, 1988; Browne, 1989; Redondo y Garrido, 1999; Smith, 1993; Wallace, 1996) ha identificado factores clínico-sanitarios (p.e., trastornos de personalidad, trastornos clínicos, alcoholismo, drogadicción), psicosociales (entre otros, disfunciones en la red social de apoyo, aprendizaje de violencia), estructurales (i.e., problemas económicos, paro) y socioculturales (v.gr., socialización en base al género, socialización en la tolerancia de la violencia sobre la mujer), como las causas de la violencia o como déficits o necesidades asociadas a los que el tratamiento debe dar respuesta para ser efectivo. Asimismo, la intervención con el penado, especialmente por tratarse de un cumplimiento en libertad, ha de velar por la función protectora de la sociedad (específicamente, de la víctima y de potenciales víctimas futuras) por lo que incluimos una medida continua de riesgo. Por último, la evaluación ha de tener presente una característica inherente a este tipo de poblaciones: el engaño, la simulación, sobresimulación o disimulación (American Psychiatric Association, 2002; Hare, Forth, y Hart, 1989). En suma, los objetivos de la evaluación son: Programar y ajustar la intervención a las necesidades, carencias y déficits de cada penado; identificar las potenciales disfunciones que puedan mitigar (e.g., limitaciones en las capacidades cognitivas) o anular (p.e., psicopatología incapacitante) la eficacia del tratamiento; aportar información para la conformación de grupos homogéneos de tratamiento; valoración de la progresión-regresión en el tratamiento de los penados; predicción del riesgo; y control del engaño, (sobre)simulación y disimulación. Por todo ello, la evaluación inicial se ejecuta por áreas y como sigue:

a) Clínica-sanitaria. En la evaluación clínica-sanitaria se dirige a la búsqueda de etiologías mentales, cognitivas o de dependencia que puedan amortiguar la eficacia o impedir el tratamiento. Para el estudio de la psicopatología se procede, acorde a las capacidades cognitivas y lingüísticas (téngase presente que un número importante de penados son extranjeros y algunos de ellos pre- sentan limitaciones en la comprensión del español $u$ otras lenguas oficiales en las Comunidades Autónomas) del penado, con las escalas clínicas básicas del MMPI-2 (Hathaway y McKinley, 1999 ) o el $S C L-90-R$ (Derogatis, 2002); el razonamiento mediante el TONI-2 (Brown, Sherbenou, y Johnsen, 1995); las conductas adictivas se miden por medio del estudio de antecedentes, la observación y registro conductual, la Entrevista Semiestructurada para Maltratadores de Género (Arce y Fariña, 2005a) y las escalas del MMPI-2, MAC-R (alcoholismo) y AAS (reconocimiento de adicción); y, de ser necesario, se lleva a cabo una exploración de las funciones neuropsicológicas mediante el Test de Evaluación Rápida de las Funciones Cognitivas (Gil, 1999) o el Test Barcelona (Peña-Casanova, 2005). Al respecto, los datos de los penados del Programa Galicia ponen de manifiesto que, en torno al $25 \%$ de los reos, hubieron de ser remitidos a un centro de deshabituación de conductas adictivas (alcoholismo, consumo de sustancias o ludopatía) de forma complementaria al tratamiento reeducativo o sustitutivo hasta la estabilización del condenado (10\%); que no llega al $2 \%$ el porcentaje de penados remitidos adicional o complementariamente a Unidades de Salud Mental para el tratamiento de enfermedades mentales que pudieran anular o mitigar la eficacia de la acción reeducativa; y que sólo 2 penados (a causa de demencia) no pudieron ser objeto de tratamiento por falta de capacidades cognitivas (otros penados con disfunciones neuropsicológicas fueron sometidos a tratamientos programados en función de las capacidades disponibles), en tanto otros con necesidades especiales pudieron ser tratados con Planes de Intervención adaptados a sus carencias cognitivas.

b) Psicosocial. La evaluación psicosocial tiene por objeto la valoración del control emocional, del autoconcepto, las estrategias de afrontamiento, de la asunción de responsabilidad, de la preparación para el cambio, la aportación de información para la confección de grupos homogéneos de tratamiento, y la identificación de las cogniciones y comportamientos desadaptados. De la Entrevista Semiestructurada para Maltratadores de Género (Arce y Fariña, 2005a) obtenemos información sociodemográfica (v.gr., edad, nivel cultural), de las relaciones sociales (p.e., apoyo social percibido y real, habilidades sociales y de comunicación); descripción de los episodios de maltrato (i.e., frecuencia, duración, intensidad, consecuencias de los episodios violentos, asunción de responsabilidad) y, motivación para el cambio e interpretación personal de su actual situación vital (entre otras, expectativas sobre el programa, punto de vista sobre los motivos de su 
conducta violenta, aceptación de la necesidad de un cambio comportamental). El control de las emociones, de las atribuciones, del autoconcepto, de las estrategias de afrontamiento y de las cogniciones distorsionadas se aprecia a través de una adaptación española de Fernández-Berrocal, Extremera, y Ramos (2004) de la Escala Rasgo de Metaconocimiento sobre Estados Emocionales; de la Escala de Locus de Control (Rotter, 1966); de las escalas de Atribución de Responsabilidad y Minimización (Lila, Herrero, y Gracia, 2008); de la Escala de Autoconcepto de Tennesse (Fitts, 1965), del Inventario de Respuestas de Afrontamiento (Moos, 1993), y del Cuestionario de Creencias Irracionales y Pensamientos Distorsionados sobre el Empleo de la Violencia, los Roles de Género y la Relación de Pareja (Arce y Fariña, 2005b).

c) Sociocultural. Los efectos de la socialización y aprendizaje de la violencia de género se indagan con el estudio del historial sociofamiliar de violencia (e.g., aprendizaje de valores y estereotipos sexistas, exposición a la violencia de género) proveniente de la Entrevista Semiestructurada para Maltratadores de Género (Arce y Fariña, 2005a).

d) Estructural. El estudio de la situación económica, laboral, vivienda, familiar (responsabilidades, hijos) se elabora a partir de fuentes documentales y de la Entrevista Semiestructurada para Maltratadores de Género (Arce y Fariña, 2005a).

e) Del riesgo. Las medidas penales asumen, como una función básica, la protección de la sociedad. Esta máxima merece una consideración especial en casos de violencia de género y en el cumplimiento de penas en libertad. Por ello es imprescindible tener una evaluación del riesgo que permita la elaboración de estrategias para gestionar dicho riesgo. Para este cometido acudimos a la observación y registro conductual, fuentes documentales, la evaluación de la salud mental y la adaptación española de Andrés-Pueyo y López (2005) de la Guía SARA (Spousal Assault Risk Assessment Guide). De este último instrumento también nos valemos, al terminar la intervención reeducativa y el seguimiento, para la formulación de un pronóstico final del riesgo de reincidencia en las conductas violentas hacia la mujer. La evaluación del riesgo, en el contexto penitenciario, no sólo ha de tener por objeto la protección de potenciales víctimas, sino también las autolesiones (en libertad, ideas e intentos autolíticos), especialmente en la fase inicial del Programa. Este riesgo se estima de la evaluación clínica, fuentes documentales, y la observación y registro conductual, definiéndose, de ser el caso, tácticas para afrontarlo. Complementariamente, también se lleva a cabo una interpretación clínica de la peligrosidad en aquellos casos que así lo aconsejen (la información se obtiene de las medidas anteriormente expuestas para la evaluación psicopatológica y de la observación y registro conductual).

f) Control de las distorsiones en las respuestas. Para el control de la validez de lo autoinformado se siguen los protocolos de Arce y Fariña (2006, 2007a; Arce, Pampillón, y Fariña, 2002) para el estudio de la (sobre)simulación y disimulación. En el estudio de los penados en el Programa Galicia (Fariña, Arce, y Novo, 2008b) hallamos (sobre)simulación en el 4\%, disimulación en el $16 \%$ y respuestas invalidadas (otra forma de simulación) en el $11 \%$. En total, controlados los casos de sobremedida, distorsionaban las respuestas de la evaluación clínica en torno al $30 \%$. Todos estos penados son objeto de un especial seguimiento sospechándose, en relación a la eficacia del tratamiento, en aquellos con (sobre)simulación y respuestas inválidas, falta de adherencia al tratamiento; y, entre los que se registra disimulación, adicción y adherencia o progreso en el tratamiento fingidos.

La evaluación del penado es continua a lo largo del programa procediéndose a una reevaluación de la evolución del penado en el tratamiento cada tres meses o al término de las unidades o módulos temáticos y a una evaluación de riesgo continuada (ésta última se relaciona con riesgos graves e inminentes de agresión de los que a fecha de diciembre de 2009 se habían emitido 64 informes que se remitieron a las Unidades contra la Violencia sobre la Mujer de las Subdelegaciones del Gobierno para que los gestionaran). De la evaluación trimestral, cuyos contenidos están circunscritos a la evaluación del riesgo y a la medida de los objetivos específicos y operativos del tratamiento al que se sometió al penado en ese período, se informa a Instituciones Penitenciarias y al Juzgado de referencia concluyendo sobre la progresión o regresión en el tratamiento del penado. Si la regresión o falta de progreso en el tratamiento se imputa a la actitud del penado, se solicita al Juzgado que tome una decisión sobre la ejecución de la pena (generalmente, y muy especialmente cuando concurre riesgo, les revocan la suspensión o sustitución). Una vez ejecutado el programa individualizado a cada penado se contrasta la efectividad de la intervención en su totalidad, esto es, en los déficits, carencias y necesidades que presentaba en la evaluación inicial. Esta evaluación sirve para dotar de contenidos la fase de seguimiento del penado de modo que se prevén, en su caso, sesiones de recuerdo o complementarias de tratamiento. En esta fase también se presta especial atención a la intervención en las áreas psicosocial (i.e., red de apoyo social) y estructural (v.gr., desempleo). Adicionalmente se reevalúa el riesgo de reincidencia fijándose, de ser necesario, unas 
estrategias de intervención sobre los factores dinámicos de riesgo registrados. Este riesgo se vuelve a evaluar una vez terminada la fase de seguimiento estableciéndose un pronóstico de reincidencia.

\section{De las líneas maestras de una intervención con penados por violencia de género en libertad}

Una vez identificados los contenidos de la intervención para cada penado en función de la evaluación, se ha de confeccionar un plan de intervención ajustado a cada uno. Éste ha de concretarse dentro del marco de una serie de condicionantes que se explicitan seguidamente.

\section{De la amplitud y longitud de la intervención}

Tras la violencia de género se esconden lo que se ha dado en denominar cogniciones tóxicas que son propias de los agresores más resistentes al tratamiento, como son los de género y sexuales. Éstas, que se caracterizan por ser internas, estables y globales (Maruna, 2004) y que subyacen a la violencia de género, discriminan entre competencia y vulnerabilidad y, en el caso que nos ocupa, se relacionan con lo que se denomina perspectiva de género, esto es, la ideología patriarcal que sustenta las cogniciones y comportamientos del agresor para ejercer poder y control sobre la mujer (i.e., actitudes, creencias y comportamientos irracionales y desadaptativos en torno al género, y al empleo de conductas violentas y desadaptativas hacia la mujer). Estas cogniciones tóxicas en torno al género dirigen la exposición social y el procesamiento cognitivo (percepción, interpretación, elaboración de los objetivos, evaluación de la respuesta, selección de la respuesta y ejecución del comportamiento) de modo que contribuyen a incrementar la vulnerabilidad o propensión de agresiones, en este caso, de género (Akhtar y Bradley, 1991; Collie, Vess, y Murdoch, 2007; Mann y Shingler, 2005), al tiempo que los convierte en especialmente resistentes al tratamiento y a no mostrar adherencia a éste (Wormith y Olver, 2002), muy especialmente cuando se manifiestan en comportamientos violentos de género (Gordon y Moriarty, 2003; Gondolf, 2000). Así pues, las intervenciones han de planificarse y ejecutarse con la suficiente amplitud. De hecho, en el contexto específico de los programas de tratamiento de maltratadores, la literatura ha informado que a mayor duración del tratamiento, menor probabilidad de futuras condenas y arrestos (p.e., Gordon y Moriarty, 2003; Gondolf, 2000). El marco legal y judicial en el que se han de desarrollar define que éstas se ejecutarán entre dos y cinco años (art. 80 del CP), siendo potestad de la jueza, juez o tribunal demarcar la duración en función de las circunstancias personales del penado, las características del hecho y la duración de la pena, de lo que se deriva que ponderan de un modo muy significativo las carencias, necesidades y riesgo observado en los penados. En consecuencia, ha de aprovecharse toda la amplitud temporal disponible porque no sólo se trata de cogniciones y subsecuentes comportamientos resistentes al tratamiento, sino que el aprendizaje e instauración de cogniciones y un repertorio comportamental alternativo no es, ni con mucho, instantáneo sino que requiere de pasos sucesivos, de ensayos continuados, de puesto en marcha de recursos para la generalización a contextos reales $\mathrm{y}$, sobre todo, para convertirlos en hábitos de comportamiento. Por el contrario, intervenciones cortas en el tiempo, aunque fueran intensas, suponen una pérdida muy significativa del potencial de la intervención porque se acortan los tiempos necesarios para la consolidación y generalización de las destrezas adquiridas y para el control de las desadaptativas. No se trata, por tanto, únicamente de dotar a la intervención de los contenidos adecuados a cada caso, sino de alcanzar una profunda interiorización de los mismos que sea impermeable al paso del tiempo y a las condiciones favorecedoras de recaídas. Una vez ejecutado el plan de intervención diseñado para el caso específico, se ha de dar cabida a un período de control, denominado seguimiento, que ha de servir para hacer un seguimiento y control del penado, planificar sesiones de recuerdo o complementarias orientadas a consolidar las destrezas adquiridas o reajustar disfunciones. En resumen, ha de aprovecharse todo el tiempo disponible para la ejecución del plan de intervención.

\section{De los niveles de intervención}

La problemática requiere que la intervención sea indefectiblemente multimodal, esto es, en las cogniciones y comportamientos (Arce y Fariña, 2007b; Beelmann y Lösel, 2006). De nada sirve la intervención en las cogniciones, tanto para el control y erradicación de las tóxicas como para el asentamiento de unas adaptativas, si no va acompañada del aprendizaje y ensayo de comportamientos alternativos. Para esto se requiere de intervenciones individualizadas sobre las cogniciones, tanto de control de las tóxicas como para el aprendizaje e instauración de las adaptativas y de comportamientos alternativos, seguidas de sesiones grupales en las que se potencian y refuerzan las cogniciones a instaurar y ejecutan comportamientos alternativos en contextos de simulación de alta fidelidad. Estas actuaciones se completan con el diseño de un plan para la generalización de lo adquirido a contextos cotidianos, valiéndonos para ello de las estrategias, acordes a cada habilidad a generalizar, que se han mostrado efectivas en tal cometido (Goldstein Sprafkin, Gershaw, y Klein, 1989; Hazel, Schumaker, Sherman, y Sheldon-Wildgen, 1981; Ollendick y Hersen, 1979; Pentz, 1980; Scott, Himadi, y Keane, 1983; Trower, Bryant, y Argyle, 1978). Ahora bien, este modelo de 
intervención no es suficiente por sí solo, ya que se dirige al nivel individual, cuando los resultados avalan, en consonancia con la propuesta de Arce y Fariña (2007b) y Farrington y Welsh (2003), la necesidad de una intervención multinivel, esto es, individual, familiar, laboral y socio-comunitaria. La intervención en los restantes niveles nuevamente estará condicionada por las circunstancias específicas de cada caso. Aún así, se han identificado dos elementos comunes: la integración laboral y la evitación de la conformación de grupos de autoayuda. Por ello, la intervención en horarios laborales convencionales no es la norma, sino la excepción, porque interfiere en la integración laboral. No en vano, la desestructuración laboral es una variable de riesgo y predictor de recaídas. Así, ha de seguirse la máxima la ejecución del plan de intervención no ha de ajustarse a los técnicos, sino éstos a los penados. Asimismo, la intervención grupal ha de ejecutarse de modo que los componentes del grupo sean desconocidos entre sí en el presente y que no puedan establecer una vinculación futura fuera del programa de intervención. La existencia o facilitación de grupos informales de maltratadores redundará, como es bien sabido, en la concreción de una especie de grupos de autoayuda o en las cogniciones tóxicas y de reforzamiento de los comportamientos desadaptativos y violentos hacia las mujeres. La no observancia de estos u otros elementos más allá del nivel individual no sólo disminuye o anula la eficacia del tratamiento, sino que puede robustecer las cogniciones que sustentan la violencia de género $\mathrm{y}$, por extensión, incrementar la probabilidad de violencia hacia las mujeres. Las actuales condiciones de implementación de los programas de intervención en torno a un CIS o recursos en las inmediaciones de la residencia, cuando no en la misma ciudad, de los maltratadores, facilita este riesgo.

\section{Control del riesgo}

La intervención con penados, y especialmente con maltratadores, ha de estar guiada siempre por el principio de máxima seguridad. Cuando la pena se cumple en condiciones de régimen cerrado, la salvaguarda de este principio está perfectamente gestionada por la prisión. No obstante, cuando la pena se ejecuta en condiciones de libertad, tal y como es el caso de las suspensiones o sustituciones de la pena, no hay un plan sistemático para la gestión de la seguridad, que, en estas condiciones, pasa a ser la de protección de la víctima original del maltratado o de otras. Los programas penitenciarios de intervención al uso se limitan a evaluar, en su caso, el riesgo inicial, sin una prescripción concreta de medidas para la gestión del riesgo. Es necesario, por tanto, concretar planes de gestión del riesgo que, además, no es estático por lo que requiere de una revisión continuada. El Programa Galicia cuenta con dos modos de control del riesgo: el ordinario y el extraordinario. El ordinario se ejecuta, conjuntamente con los informes de progresión/adherencia al tratamiento tras cada módulo unidad temática, que viene a coincidir con cada tres meses. En éste se establece el nivel de riesgo y las medidas de gestión del mismo, remitiéndose al Juzgado Sentenciador junto, cuando procede, con la recomendación de medidas judiciales a tomar. Adicionalmente, se emiten informes extraordinarios ante la posibilidad de riesgo inminente, proponiéndose medidas a las autoridades judiciales y administrativas competentes.

\section{De la adherencia y progreso en el tratamiento}

Como referimos previamente, este tipo de penados son especialmente resistentes al tratamiento por lo que la adherencia al mismo es crítica. Dos son las problemáticas relativas a este punto. Una es la resistencia inicial al tratamiento, manifestada en la no asunción de los hechos y de la responsabilidad inherente. Éste es un eje central en el tratamiento, de modo que no puede iniciar los contenidos en sí, sin que el penado asuma los hechos y su responsabilidad en éstos. Si bien mayoritariamente los maltratadores son inicialmente resistentes al tratamiento, hay un grupo significativo que recurre a dos estrategias diferentes para no adherirse realmente: simulación de una incapacidad y simulación de adherencia al tratamiento (simulación positiva). Los primeros datos cifran la suma de estas dos contingencias en torno al $30 \%$, cifra concordante con la hallada en otros contextos (e.g., Baer y Miller, 2002), lo que implica que, en aproximadamente $1 / 3$ de los penados, un plan de intervención que no gestione y evalúe efectivamente la adherencia fracasará en el objetivo general del tratamiento: la sustitución de las cogniciones tóxicas y comportamientos violentos hacia la mujer por otros adaptativos y alternativos. Evaluada y constatada la adherencia, se ha de dar inicio al tratamiento en sí, pasando a ser el objeto de la evaluación el progreso en el tratamiento. Pero de nada sirve una adherencia inicial, si no se logran los objetivos de cada uno de los módulos. Como quiera que para que una intervención sea eficaz requiere de un programa bien estructurado (McGuire, Mason, y O'Kane, 2000), la secuencia de los contenidos no puede ser aleatoria ya que el logro de los objetivos de las intervenciones, de cada módulo supone haber alcanzado los anteriores de la secuencia. Así, un penado no puede avanzar de un módulo a otro si no ha alcanzado los objetivos del primero, porque el subsiguiente requiere de las competencias marcadas como objetivo en el previo. De hecho, el progreso con éxito en el tratamiento reduce la probabilidad de futuras condenas y arrestos (Gordon y Moriarty, 2003). Por el contrario, un salto de módulo improcedente anuncia el fracaso en el programa por el encadenamiento de los objetivos. Por ello, es necesaria una evaluación continuada del logro 
e instauración de los objetivos propuestos con tres niveles de evaluación: progresión y regresión (art. 65 de la L.O. General Penitenciaria), al que es preciso añadir la categoría de estancamiento propia de aquellos casos con dificultades de aprendizaje. Esta particularidad nos lleva nuevamente a la pertinencia de una intervención prolongada en el tiempo en la que no sólo se verifique el logro puntual de los objetivos específicos, sino también el encadenamiento de objetivos y la persistencia de los logros alcanzados. Como consecuencia, es preciso que, además de la evaluación contigua de los objetivos de cada módulo, se lleven a cabo evaluaciones sistemáticas del progreso, regreso o estancamiento con una periodicidad que someta a contraste la hipótesis del encadenamiento de objetivos y de la persistencia de los logros alcanzados. La cronología de esta evaluación puede definirse por tiempos, tal y como la operativiza la L.O. General Penitenciaria, o por contenidos. Por tiempos, dado que la moda de tiempo de condena son dos años, una evaluación con una cadencia superior a los tres meses sería inoperativa, en cuanto que por contenidos debería ajustarse a las unidades temáticas que conformen los módulos.

\section{De la filosofía subyacente a la intervención}

La inmensa mayoría de los programas de intervención con maltratadores recurren a una filosofía de tratamiento terapéutica. Ésta tiene su origen en el tratamiento voluntario al que se sometían en busca de ayuda los maltratadores previamente a la entrada en vigor leyes específicas contra la violencia de género. Como es obvio, éstos buscaban entre los profesionales de ayuda, especialmente psicólogos clínicos, pero no sólo, apoyo, prestándoles éstos asistencia terapéutica. La adopción de una perspectiva terapéutica es totalmente errada cuando se trata del cumplimiento de una condena judicial. Esto por varios motivos. A la intervención terapéutica subyace el concepto de enfermedad, cuando los penados no son tales por ser enfermos, sino por un comportamiento penalizado en el código penal. Tomar a los maltratadores como enfermos supone que la responsabilidad es exógena lo que entorpece el tratamiento y facilita la recaída (i.e., Maruna y Copes, 2005; Peterson y Leigh, 1990; Werner, 1989), ya que inhibe la responsabilidad en el cambio de comportamientos futuros (Bovens, 1998) y dificulta una adecuada orientación y resolución de los problemas, esto es, en las funciones ejecutivas (Chang y D'Zurilla, 1996). Asimismo, la perspectiva terapéutica presupone que el técnico ha de prestar un servicio al penado, cuando a quien se lo presta es indirectamente a la sociedad y a la víctima real y potenciales. La prestación de un servicio implica que el técnico ha de estar a disposición del penado, cuando es el penado el que está obligado a seguir el tratamiento prescrito por el técnico y de no seguirlo será objeto de revocación de la suspensión de la condena. La asunción de un rol de penado con obligaciones y normas de comportamiento y cumplimiento (todas éstas están recogidas en el Reglamento del Programa Galicia, que puede solicitarse a los autores, incluida la de adherencia y progresión en el tratamiento, junto con las sanciones asociadas al incumplimiento), frente a la de cliente o usuario que difícilmente asume obligaciones y se implica en la consecución de objetivos porque la técnica de tratamiento que se le aplica es de ayuda.

\section{Contenidos}

No es posible establecer unos contenidos únicos, pero sí unos mínimos y relatar los más comunes en función de la consistencia teórica y de la experiencia derivada de los casos evaluados en el Programa Galicia de Reeducación de Maltratadores de Género. En consonancia con nuestro ordenamiento jurídico, el programa se ha de iniciar con la presentación al penado del programa, el reglamento y las obligaciones al que puede acceder tras solicitud de entrada en suspensión o sustitución de la pena; seguidamente se pasa a la evaluación de las características del penado; continuando la intervención con la asunción de los hechos y de la responsabilidad; y también ha de llevar contenidos de género. Los demás contenidos son específicos de cada caso. Aún así, los más preeminentes incluyen contenidos de competencia social, control de celos; y adquisición de comportamientos alternativos a la agresión (el lector interesado puede ver los contenidos más frecuentes, así como las técnicas más comunes del Programa Galicia en Arce y Fariña, 2007c).

\section{Referencias}

Akhtar, N., y Bradley, E. J. (1991). Social information processing deficits of aggressive children: Present findings and implications for social skills training. Clinical Psychology Review, 11, 621-644.

American Psychiatric Association (2002). DSM-IV-TR. Manual diagnóstico y estadístico de los trastornos mentales. Barcelona: Masson.

Andrés-Pueyo, A., y López, S. (2005). S.A.R.A. Manual para la valoración del riesgo de violencia contra la pareja. Barcelona: Publicaccions i Edicions de la Universitat de Barcelona.

Andrews, D. A., y Bonta, J. (2006). The psychology of criminal conduct (4 ${ }^{\mathrm{a}}$. ed.). Cincinnati, $\mathrm{OH}$ : Anderson Publishing Co.

Arce, R., y Fariña, F. (1996). From jurors to jury decision making. A non model approach. En G. Davis, M. McMurran, C. Wilson, y S. Lloyd-Bostock (Eds.), Psychology, law and criminal justice. International developments in research and practice (pp. 337-343). Berlin: Walter de Gruyter. 
Arce, R., y Fariña, F. (2005a). Entrevista semiestructurada para maltratadores de género. Manuscrito no publicado. Unidad de Psicología Forense, Universidad de Santiago de Compostela.

Arce, R., y Fariña, F. (2005b). Cuestionario de creencias irracionales y pensamientos distorsionados sobre el empleo de la violencia, los roles de género y la relación de pareja (dependencia emocional). Manuscrito no publicado. Unidad de Psicología Forense, Universidad de Santiago de Compostela.

Arce, R., y Fariña, F. (2006). Construcción y validación de un protocolo para la evaluación forense de la disimulación. Manuscrito no publicado. Unidad de Psicología Forense, Universidad de Santiago de Compostela.

Arce, R., y Fariña, F. (2007a). Propuesta de un protocolo válido y fiable para la evaluación psicológico-forense de la imputabilidad. En R. Arce, F. Fariña, E. Alfaro, C. Civera y F. Tortosa. (Eds.), Psicología jurídica. Evaluación e intervención (pp. 59-65) Valencia: Servicio de Publicaciones de la Diputació de València.

Arce, R., y Fariña, F. (2007b). Teorías de riesgo de la delincuencia. Una propuesta integradora. En F. J. Rodríguez y C. Becedóniz (Coords.), El menor infractor Posicionamientos y realidades (pp. 37-46). Oviedo: Servicio de Publicaciones del Principado de Asturias.

Arce, R., y Fariña, F. (2007c). Intervención psicosocial con maltratadores de género. En J. M. Sabucedo y J. Sanmartín. Los escenarios de la violencia (pp. 29-43). Barcelona: Ariel.

Arce, R., Fariña, F., Seijo, D., Novo, M., y Vázquez, M. J. (2005). Contrastando los factores de de riesgo y protectores del comportamiento inadaptado en menores: Implicaciones para la prevención. En Centro de Investigación y Documentación Educativa (Ed.), Premios nacionales de investigación educativa 2004 (pp. 17-50). Madrid: Ministerio de Educación y Ciencia.

Arce, R., Pampillón, M. C., y Fariña, F. (2002). Desarrollo y evaluación de un procedimiento empírico para la detección de la simulación de enajenación mental en el contexto legal. Anuario de Psicología, 33, 385-408.

Arce, R., Seijo D., Fariña, F., y Novo, M. (2004). Family interaction factors: Analyzing their effects on personal, social and school inadaptability, and antisocial and delinquent behavior. En A. Czerederecka, T. JaskiewicObydzinska, R. Roesch y J. Wójcikiewicz (Eds.), Forensic psychology and law. Facing the challenges of a changing world (pp. 435-441). Kraków: Institute of Forensic Research Publishers.

Babcok, J. C., Green, C. E., y Robie, C. (2004). Does batterers' treatment work? A meta-analytic review of domestic violence treatment. Clinical Psychology Review, 23, 1023-1053.

Baer, R. A., y Miller, J. (2002). Underreporting of psychopathology on the MMPI-2: A meta-analytic review. Psychological Assessment, 14, 16-26.

Bartol, C. R. (1999). Criminal behavior. A psychosocial approach ( $5^{\mathrm{a}}$ ed.). Upper Saddle River, NJ: Prentice Hall.

Beelmann, A., y Lösel, F. (2006). Child social skills training in developmental crime prevention: Effects on antisocial behavior and social competence. Psicothema, 18, 603610.

Beleña, M. A., y Báguena, M. J. (1993). Nivel de reincidencia y diferencias individuales en motivación e inteligencia en mujeres delincuentes. En M. García (Comp.), Psicología social aplicada en los procesos jurídicos y políticos (pp. 145-151). Sevilla: Eudema.

Bersani, C. A., y Chen, H. (1988). Sociological perspectives in family violence. En V. B. van Hasselt, R. L. Morrison, A. S. Bellack, y M. Hersen (Eds.), Handbook of family violence (pp. 57-88). Nueva York: Plenum Press.

Bonta, J., Law, M., y Hanson, K. (1998). The prediction of criminal and violent recidivism amongst mentally disordered offenders: A meta-analysis. Psychological Bulletin, 123, 123-142.

Bovens, M. (1998). The quest for responsibility: Accountability and citizenship in complex organisations. Cambridge, UK: Cambridge University Press.

Bowen, E. Gilchrist, E., y Beech, A. R. (2008). Change in treatment has no relationship with subsequent re-offending in U.K. domestic violence sample: A preliminary study. International Journal of Offender Therapy and Comparative Criminology, 52, 598-614.

Brown, L., Sherbenou, R., y Johnsen, S. (1995). Toni-2. Madrid: TEA Ediciones.

Browne, K. D. (1989). Family violence: Spouse and elder abuse. En K. Howells y C. R. Hollin (Eds.), Clinical approaches to violence (pp. 119-154) Nueva York: John Wiley \& Sons.

Carney, M. M., y Buttell, F. A. (2004). A multidimensional evaluation of a treatment program for female batterers: A pilot study. Research on Social Work Practice, 14, 249258.

Chang, E. C., y D'Zurilla, T. J. (1996). Relations between problem orientation and optimism, pessimism, and trait affectivity: A construct validation study. Behaviour Research and Therapy, 34, 185-194.

Collie, R. M., Vess, J., y Murdoch, S. (2007). Violence-related cognition: Current research. En T. A. Gannon, T. Ward, A. R. Beech, y D. Fisher (Eds.), Aggressive offenders' cognition. Theory, research, and practice (pp. 179-197). Chichester: John Wiley and Sons.

Derogatis, L. R. (2002). SCL-90-R. Manual. Madrid: TEA Ediciones.

D'Zurilla, T. J. (1986). Problem-solving therapy. A social competence approach to clinical interventions. Nueva York: Springer Verlag.

Echeburúa, E., Corral, P., Fernández-Montalvo, J., y Amor, P. J. (2004). ¿Se puede y debe tratar psicológicamente a los hombres violentos contra la pareja? Papeles del Psicólogo, 88, 10-18.

Echeburúa, E., y Fernández-Montalvo, J. (1997). Tratamiento cognitivo-conductual de hombres violentos en el hogar: Un estudio piloto. Análisis y Modificación de Conducta, 23, 355-384.

Echeburúa, E., y Fernández-Montalvo, J. (1998). Hombres maltratadores. En E. Echeburúa y P. Corral (Eds.), 
Manual de Violencia Familiar (pp. 73-175). Madrid: Editorial Siglo XXI.

Echeburúa, E. Fernández-Montalvo, J., y Corral, P. (2008). ¿Hay diferencias entre la violencia grave y la violencia menos grave contra la pareja? Un análisis comparativo. International Journal of Clinical and Health Psychology, 8, 355-382.

Fariña, F., y Arce, R. (2003). Avances en torno al comportamiento antisocial, evaluación y tratamiento. Madrid: Ministerio de Trabajo y Asuntos Sociales.

Fariña, F., Arce, R., y Novo, M. (2008). Estado clínico y tratamiento penitenciario en penados en tercer grado por violencia de género. En F. J. Rodríguez, C. Bringas, F. Fariña, R. Arce, y A. Bernardo (Eds.), Psicología jurídica. Entorno judicial y delincuencia (pp. 375-382) Oviedo: Ediciones de la Universidad de Oviedo.

Fariña, F., Arce, R., y Novo, M. (2008). Neighborhood and community factors: Effects on deviant behavior and social competence. The Spanish Journal of Psychology, 11, 78-84.

Farrington, D. P. (1992). Explaining the beginning, progress and ending of antisocial behavior from birth to adulthood. En J. McCord (Ed.), Facts, frameworks and forecasts: Vol. 3. Advances in criminological theory (pp. 253-268). New Brunswick, Canada: Transaction Publishers.

Farrington, D. P. (1996). Understanding and preventing youth crime. Nueva York: Joseph Rowntree Foundation.

Farrington, D. P. (2000). Psychological predictors of adult antisocial personality and adult convictions. Behavioural Sciences and the Law, 18, 605-622.

Farrington, D. P. (2003). Advancing knowledge about the early prevention of adult antisocial behavior. En D. P. Farrington y J. W. Coid (Eds.), Early prevention of antisocial behavior (pp. 1-31). Cambridge: Cambridge University Press.

Farrington, D. P., y Welsh, B. C. (2003). Family-based prevention of offending: a meta-analysis. Australian and New Zeeland Journal of Criminology, 36, 127-151.

Fernández-Berrocal P., Extremera N., y Ramos, N. (2004). Validity and reliability of the Spanish modified version of the Trait Meta-Mood Scale. Psychological Reports, 94, 751-755.

Feldman, M. P. (1989). Comportamiento criminal: Un análisis psicológico. México: Fondo de Cultura Económica.

Fitts, W. H. (1965). Tennessee self concept scale. Counsellor recordings and test. Manual., Tennessee: Nashville.

Fracher, J. C. (2003). Changing violent man. Journal of Psychiatry and Law, 31, 99-101.

Garrido, V., y López, M. J. (1995). La prevención de la delincuencia: El enfoque de la competencia social. Valencia: Tirant lo Blanch.

Gil, R. (1999). Neuropsicología. Barcelona: Masson.

Goldstein, A. P., Sprafkin, R. P., Gershaw, N. J., y Klein, P. (1989). Habilidades sociales y autocontrol en la adolescencia. Un programa de enseñanza. Barcelona: Martínez Roca.

Gondolf, E. W. (2000). A 30-mounh follow-up of courtreferred batterers in four cities. International Journal of
Offender Therapy and Comparative Criminology, 44, 111-128.

Gondolf, E. W. (2004). Evaluating batterer counseling programs: A difficult task showing some effects and implications. Aggression and Violent Behavior, 9, 605-63.

Gondolf, E. W., y White, R. J. (2001). Batterer program participants who repeatedly reassault: Psychopathic tendencies and other disorders. Journal of Interpersonal Violence, 16, 361-380.

Gordon, J. A., y Moriarty, L. J. (2003). The effects of domestic violence batterer treatment on domestic violence recidivism: The Chesterfield County experience. Criminal Justice and Behavior, 30, 118-134.

Gottfredson, M. R., y Hirschi, T. (1990). A general theory of crime. Stanford, CA.: Stanford University Press.

Gracia, E., Herrero, J., Lila, M., y Fuente, A. (2009). Perceived neighborhood social disorder and attitudes toward domestic violence against women among LatinAmerican immigrants. The European Journal of Psychology Applied to Legal Context, 1, 25-43.

Hare, R. D., Forth, A. E., y Hart, S. D. (1989). The psychopath as prototype for pathological lying and deception. En J. C. Yuille (Ed.), Credibility assessment (pp. 2549). Dordrecht: Kluwer.

Hathaway, S. R., y Mckinley, J. C. (1999). MMPI-2. Inventario multifásico de personalidad de Minnesota-2. Manual. Madrid: TEA.

Hazel, J. S., Schumaker, J. B., Sherman, J. A., y SheldonWildgen, J. (1981). The development and evaluation of a group skills training program for court-adjudicated youths. En D. Upper y S. M. Ross (Eds.), Behavioral group therapy, 1981: An annual review (pp. 113-152). Champaign, IL: Research Press Company.

Holtzworth-Munroe, A., Meehan, J. C., Herron, K., Rehman, U., y Stuart, G. L. (2000). Testing the HoltzworthMunroe and Stuart (1994) batterer typology. Journal of Consulting and Clinical Psychology, 68, 1000-1019.

Holtzworth-Munroe, A., y Stuart, G. L. (2004). Typologies of male batterers: Three subtypes and differences among them. Psychological Bulletin, 116, 476-497.

Lila, M., Herrero, J., y Gracia, E. (2008). Atribución de responsabilidad y minimización en hombres penados por violencia contra la mujer: Un instrumento de evaluación. En F. J. Rodríguez, C. Bringas, F. Fariña, R. Arce y A. Bernardo (Eds.), Psicología jurídica. Familia y victimología (pp. 271-279). Oviedo: Ediciones de la Universidad de Oviedo.

Loinaz, I., Torrubia, R., Echeburúa, E., Navarro, J. C., Fernández, L. (2009). Implicaciones de las tipologías de agresores para el tratamiento en prisión. Cuadernos de Medicina Psicosomática y Psiquiatría de Enlace, 91, 19-25.

López, M. J., Garrido, V., y Ross, R. R. (2001). El programa del pensamiento prosocial: Avances recientes. Valencia: Tirant lo Blanch.

Lösel, F., y Bender, D. (2003). Protective factors and resilience. En D. P. Farrington y J. W. Coid (Eds.), Early prevention of antisocial behavior (pp. 130-204). Cambridge: Cambridge University Press. 
Lösel, F., Kolip, P., y Bender, D. (1992). Stress-resistance in a multiproblem milieu: Are resilient juveniles 'Superkids'. Zeitschrift für Klinische Psychologie, 21, 48-63.

Mann, R. E., y Shingler, J. (2005). Schema-driven cognition in sexual offenders: Theory, assessment and treatment. En W. L. Marshall, Y. M. Fernández, L. E. Marshall, y G. A. Serran (Eds.), Sexual offender treatment: Controversial issues (pp. 173-186). Chichester: John Wiley and Sons.

Maruna, S. (2004). Desistance and explanatory style: a new direction in the psychology of reform. Journal of Contemporary Criminal Justice, 20, 184-200.

Maruna, S., y Copes, H. (2005). What have we learned in five decades of neutralization research? Crime and Justice: A Review o Research, 32, 221-320.

McGuire, J. (2000). Explanations of criminal behaviour. En J. McGuire, T. Mason y A. O'Kane (Eds.), Behavior, crime and legal processes. A guide for forensic practitioners (pp. 135-159). Chichester: John Wiley and Sons.

McGuire, J., Mason, T., y O'Kane, A. (2000). Effective interventions, service and policy implications. En J. McGuire, T. Mason y A. O'Kane (Eds.), Behavior, crime and legal processes. A guide for forensic practitioners (pp. 289-314). Chichester: John Wiley and Sons.

Ministerio de Igualdad (2009). Balance de las denuncias y homicidios por violencia machista registrados en 2008. Recuperado el 31 de Enero de 2009, de http://www.observatorioviolencia.org/documentos.php?id=202

Moffitt, T. E. (1993). Adolescence-limited and life-coursepersistent antisocial behavior: A developmental taxonomy. Psychological Review, 100, 674-701.

Moos, R. (1993). Coping response inventory adult form-professional. Manual. Florida: Psychological Assessment Resources, Inc.

Ollendick, T. H., y Hersen, M. (1979). Social skills training for juvenile delinquents. Behaviour Research and Therapy, 17, 547-554.

Pentz, M. A. (1980). Assertion training effects on unassertive and aggressive adolescents. Journal of Counselling Psychology, 27, 76-83.

Peña-Casanova, J. (2005). Test Barcelona-R. Barcelona: Masson.

Peterson, G. W., y Leigh, G. K. (1990). The family and social competence in adolescence. En T. P. Gullotta, G. R. Adams, y R. Montemayor (Eds.), Developing social competence in adolescence. Advances in adolescent development (Vol. 3, pp. 97-139). Thousand Oaks, CA: Sage.

Puffet, N. K., y Gavin, C. (2004). Predictors of program outcome and recidivism at the Bronx Misdemeanor Domestic Violence Court. Nueva York: Center for Court Innovation.
Redondo, S., y Garrido, V. (1999). Propuesta para la el tratamiento en la comunidad de los agresores intrafamiliares. Recuperado el 31 de Enero de 2009, de http://www.ub.edu/geav/Imatges/MALTRATADORESFAMILIARES.pdf

Ross, R. R., y Fabiano, E. A. (1985). Time to think: A cognitive model of delinquency prevention and offender rehabilitation. Johnson City, Tenn.: Institute of Social Sciences and Arts Inc.

Rotter J. B. (1966). Generalized expectancies for internal versus external control of reinforcement. Psychological Monographs, 80 (1, Whole No. 609).

Scott, R. R., Himadi, W., y Keane, T. M. (1983). A review of generalization in social skills training: Suggestions for future research. En M. Hersen, H. M. Eisler, y P. M. Miller (Eds.), Progress in behaviour modification (Vol. 15, pp. 114-172). Nueva York: Academic Press.

Smith, L. J. (1993). Domestic violence: An overview of the literature. Londres: HMSO.

Taylor, B., Davis, R., y Maxwell, C. (2001). The effects of a group batterer programme: A randomized experiment in Brooklyn. Justice Quarterly, 18, 170-201.

Trower, P., Bryant, B., y Argyle, M. (1978). Social skills and mental health. Londres: Methuen.

United Nations (1988). Committee on crime prevention and control. Report on the tenth session. Viena: United Nations Publications.

Ventura, L. A., y Davis, G. (2005). Domestic violence: Court case conviction and recidivism. Violence against Women, $11,255-277$.

Wallace, H. (1996). Family violence: Legal, medical, and social perspectives. Boston: Allyn and Bacon.

Wallston, K. A. (1992). Hocus-pocus, the focus isn't strictly on locus: Rotter's social learning theory modified for health. Cognitive Therapy and Research, 16, 183199.

Werner, E. E. (1986). The concept of risk from a developmental perspective. Advances in Special Education, 5, 123.

Werner, E. E. (1989). High-risk children in young adulthood: A longitudinal study from birth to 32 years. American Journal of Orthopsychiatry, 59, 72-81.

Wormith, J. S., y Olver, M. E. (2002). Offender treatment attrition and its relationship with risk, responsivity and recidivism. Criminal Justice and Behavior. Special Issue: Risk and assessment in contemporary corrections, 29, 447-471.

Zubin, J. (1989). Suiting therapeutic intervention to the scientific models of aetiology. British Journal of Psychiatry, $115,9-14$

Manuscrito Recibido: 12/03/2010

Revisión Recibida: 07/06/2010

Manuscrito Aceptado: 07/06/2010 